\title{
Tunable Bandpass Filter with Two Transmission Zeros
}

\author{
Zhongyin Xiao ${ }^{a}$, Xiyang $\mathrm{Xu}^{\mathrm{b}}$ \\ School of Communication and Information Engineering, Shanghai University, Shanghai 200444, \\ China. \\ azhyxiao@shu.edu.cn, bxxyzx1994@outlook.com
}

\begin{abstract}
In this paper, a novel varactor-tuned microstrip bandpass filter consisting of two dualmode open loop resonators was proposed. The two open loop resonators are placed diagonally to prevent the occurrence of resonance between them. No coupling exists between the operating oddmode and even-mode. Based on this feature, we use a simple DC bias circuit to tune the odd and even mode frequency of the resonator respectively to realize tunable passband frequency. The results show that two transmission zeros are produced at the edge of each passband, which greatly improves the frequency selectivity of the filter. This filter is tuned from $0.87 \mathrm{GHz}$ to $1.03 \mathrm{GHz}$ with return loss more than $20 \mathrm{~dB}$ with nearly constant frequency response.
\end{abstract}

Keywords: Tunable filter, dual-mode resonator, bandpass filter, even-odd mode.

\section{Introduction}

With the rapid development of wireless communication systems, spectrum congestion will be more striking. In order to improve the utilization of spectrum resources, multi-passband, dynamic frequency allocation and frequency hopping spread spectrum technology are widely used, which greatly facilitate the electrical transfer or reconfigurable microwave device (such as filters) development [1-3].

Tunable filters may be designed based on single or dual-mode resonators, and preferably in microstrip, on which dc bias circuits are easily implemented. In recent years, people are more interested in the resonant mode that each dual mode microstrip resonator may be used as a double resonant circuit, therefore, the number of resonators required for a given degree of filter is reduced by half, resulting in a compact filter configuration. For a conventional dual-mode filter, the two degenerate modes are coupled by controlling an appropriate perturbation, such as the loop filter[4], the square loop filter[5], and the stepped impedance ring filter[6]. On the other hand, a new type of dual-mode resonator filter based on a triangular patch has been investigated [7], for which the dual modes do not couple. More recently, this unique characteristic has been demonstrated again with a miniature dual-mode microstrip open-loop resonator [8], which is improved from the conventional single-mode open-loop resonator [9]. The passband insertion loss of tunable filter[10] is too high, and this filter only produces a transmission zero, whose selectivity is not good. The tunable filter[11] increased the tuning range by adding complex external circuit, while this paper only by changing size of minor L. In order to simplify the the design and to further improve the performance of the filter, in this paper, we present an investigation of a new type of miniature microstrip dual-mode resonator for filter applications. The proposed new dual-mode resonator is developed from a singlemode (operated) open-loop resonator [8]. And then we analyze the couple chacteristic of individual resonators, two transmission zeros are produced at the edge of passband, and the filter finally achieve a high bandpass performance.

\section{Dual-Mode Open-Loop Resonator and Filter}

Dual-mode open-loop resonator and filter are shown in Fig.1. The open-loop mode resonator shown in Fig.1 (a), which is composed of an open square ring resonator and a plus in the middle of the T-open stub lines. The open-loop mode resonant filter shown in Fig.1(b), the resonator coupled with the input-output ports through the slit. Owing to the symmetric structure, the resonant characteristic of the resonator can be analyzed by the odd-even mode method. The odd-mode equivalent circuit is shown in Fig. 2. 


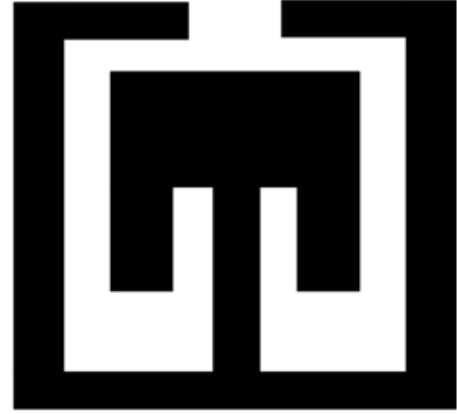

(a)

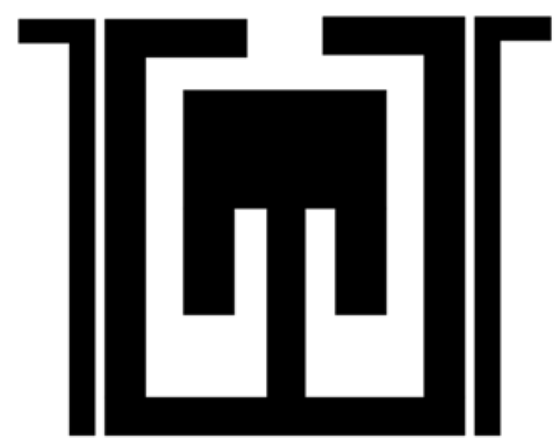

(b)

Fig 1. Dual-mode open-loop resonator and filter (a) Resonator, (b) Filter

For the odd mode, the input admittance can expressed as:

$$
Y_{\text {in }, \text { odd }}=-j Y_{o} \cot \theta_{o}
$$

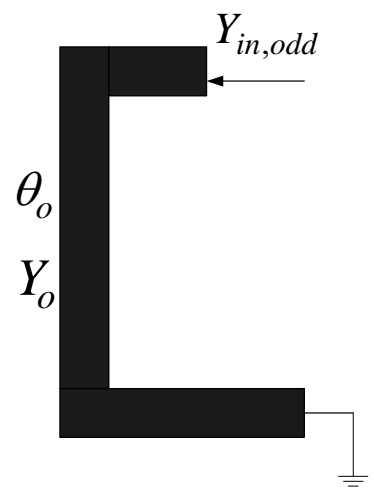

(a)

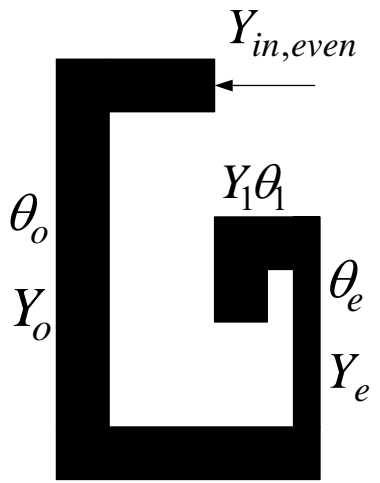

(b)

Fig 2. Circuit models. (a) Odd mode. (b) Even mode

When the resonance occurs, $Y_{\text {in,odd }}=0$, the odd mode resonant frequency can be calculated; For the even mode, the input admittance can be written as:

$$
Y_{\text {in,even }}=j Y_{o} \frac{Y_{L}+Y_{o} \tan \theta_{o}}{Y_{o}-Y_{L} \tan \theta_{o}}
$$

With:

$$
Y_{L}=Y_{e} \tan \theta_{e}+Y_{1} \tan \theta_{1}
$$

When the resonance occurs, $Y_{\text {ineven }}=0$, therefore, the even mode resonant frequency can be calculated.

Here, $\theta_{o}$ and $\theta_{e}$ denote the electrical length of the microstrip line in Figure 2, while $Y_{o}$ is the openloop microstrip line admittance.

According to the dual resonance model, we can see the input and output through the odd and even mode coupling, while the odd mode and even mode do not couple to each other. Coupling matrix of the model is as follows[12]:

$$
M=\left[\begin{array}{cccc}
0 & M_{S o} & M_{S e} & 0 \\
M_{S o} & M_{o o} & 0 & M_{S e} \\
M_{S e} & 0 & M_{e e} & -M_{S o} \\
0 & M_{S e} & -M_{S e} & 0
\end{array}\right]
$$

where $M_{i j}(\mathrm{i}=\mathrm{s}, \mathrm{e}, \mathrm{o} ; \mathrm{j}=\mathrm{e}, \mathrm{o})$ denotes the coupling coefficient between $\mathrm{i}$ and $\mathrm{j}, \mathrm{s}$ denotes source, e denotes odd-mode, and o denotes even-mode. From the theoretical analysis of the coupling matrix, 
the filter will produce a transmission zero. The transmission zero lowpass prototype of normalized frequency can be expressed as:

$$
\Omega=\frac{M_{o o} M_{S e}^{2}-M_{e e} M_{S o}^{2}}{M_{S e}^{2}-M_{S o}^{2}}
$$

When $\Omega>0$, transmission zero is in the high end of the passband and when $\Omega<0$, transmission zero is the lower end of the passband.

Where:

$$
\begin{gathered}
M_{e e}=2\left(f_{0}-f_{e}\right) / \Delta f \\
M_{o o}=2\left(f_{0}-f_{o}\right) / \Delta f
\end{gathered}
$$

Here, $f_{0}$ is the passband center frequency, $f_{o}$ and $f_{e}$ are the odd mode and even mode resonant frequency, $\Delta f$ is passband bandwidth.

Theoretical analysis shows that a filter will produce a transmission zero and can be designed to produce at high end or low end. The two cascaded filters can produce a transmission zero in low end and high end, respectively. Adjusting the resonator in the middle of T-type structure can change the width of the transmission zero position. To prevent the occurrence of resonance between the two filters, the two filters placed diagonally, the model shown in Figure 3.

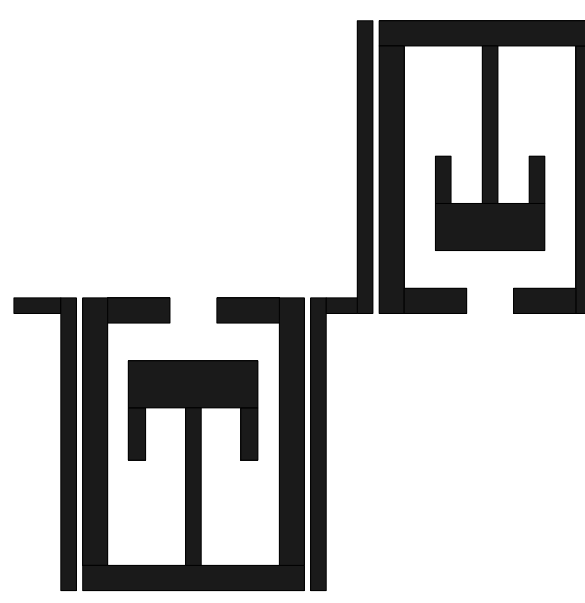

Fig 3. Layout of the filter

Through the above analysis, we designed microstrip filter structure shown in Figure 3, which uses two open-loop mode resonator. The simulation results can be seen in Figure 4. The design of microstrip filter achieves the expected results, while generating two transmission zeros, steep passband edge selectivity, and in-band loss is less than $3 \mathrm{~dB}$.

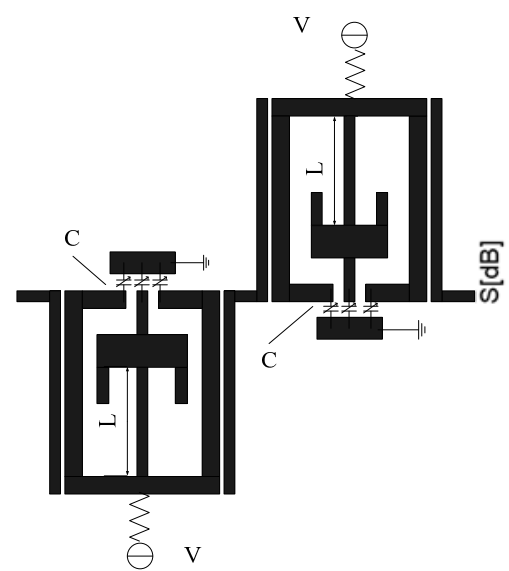

Fig 5. A layout of the proposed filter

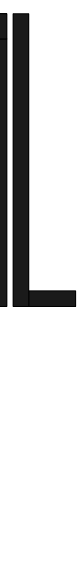

Fig 4. Simulated performance of the filter
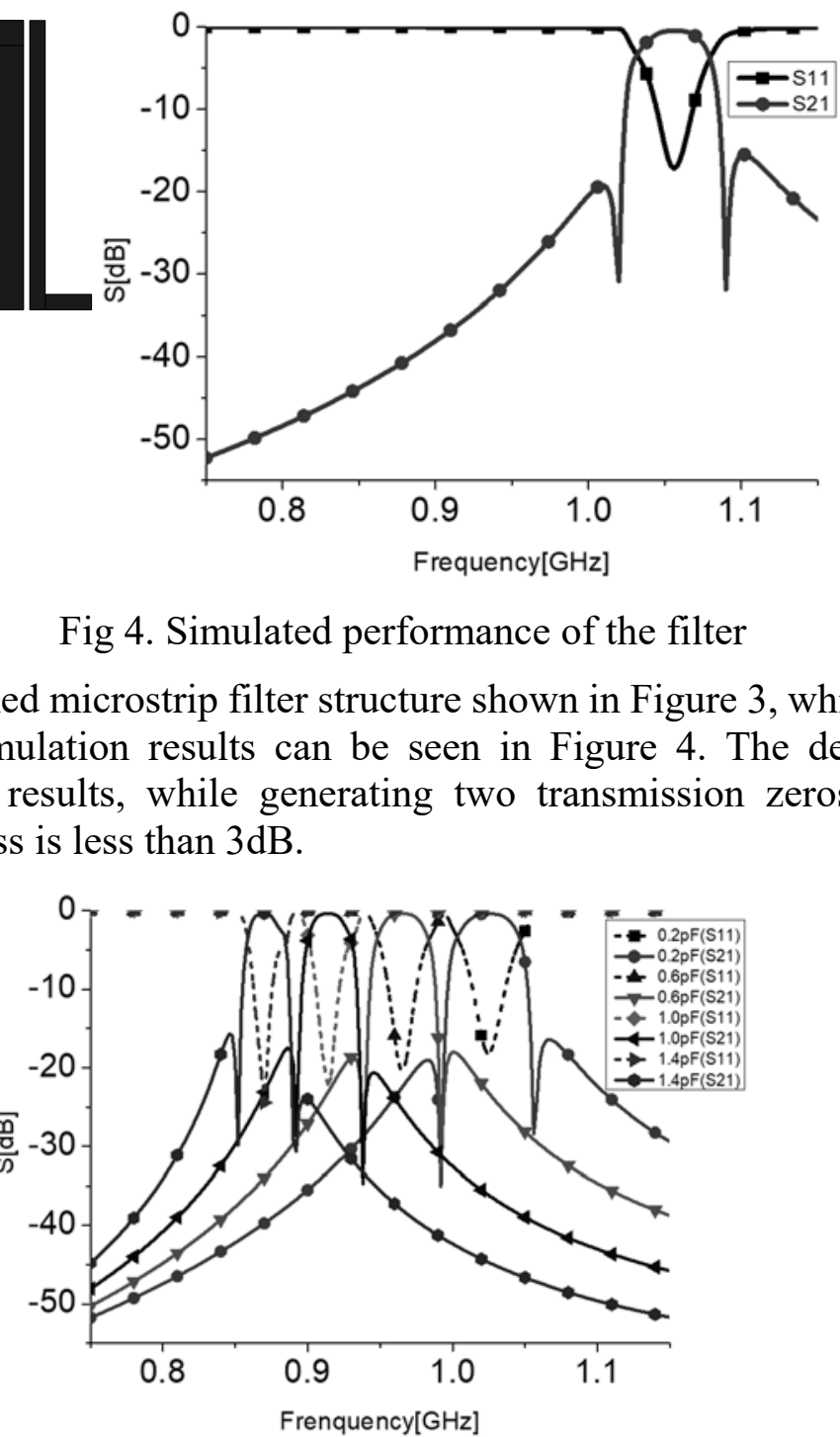

Fig 6. Simulated performance of the filter 
Figure 5 is the design of the tunable filter, $\mathrm{C}$ is the load on the filter varactor, and six varactors work through a DC bias voltage $\mathrm{V}$. Changing the width and length of $\mathrm{L}$, the tuning range can be improved. When $\mathrm{L}$ is longer and thinner, with the change in capacitance, odd-mode resonator appears apparent frequency shift. By adjusting the size of $\mathrm{L}$, the odd mode and even mode frequency show consistent frequency deviation, which can effectively adjust the filter's passband frequency and bandwidth. Tunning the voltage of varactor diode can change the capacitance values. The simulation results shown in Figure 6, can be seen that the filter response curve of elliptic functions, and the capacitor tunes from $0.2 \mathrm{pf}$ to $1.4 \mathrm{pf}$ while center frequency changes from $0.87 \mathrm{GHz}$ to $1.03 \mathrm{GHz}$. When passband changes, the response curve shape is consistent with the insertion loss less than $3 \mathrm{~dB}$, two transmission zeros close to the pass band edge selectivity. What is more, the absolute bandwidth becomes wider when the capacitance becomes large.

\section{Summary}

In summary, we have designed a novel tunable bandpass filter with two transmission zeros. Two varactor diodes are put at the open end of an open loop to adjust the resonant frequency of the odd mode, while the other varactor diode is minor and put at the center of the end of the $T$ to adjust the even mode frequency. The center frequency of the filter is adjustable with range from $0.87 \mathrm{GHz}$ to $1.03 \mathrm{GHz}$ and return loss is greater than $20 \mathrm{~dB}$. Simulation results show that two transmission zeros close to the passband edge which greatly improves the frequency selectivity of the filter. The filter can find its application in a mobile communication system for GSM 900MHz.

\section{References}

[1]. R.Arslanalp, E.Yuce, A.Tola. Two lossy integrator loop based current-mode electronically tunable universal filter employing only grounded capacitors. Microelectronics Journal. Vol.59 (2017) No.1, p.1-9.

[2]. H. Zhu, A. Abbosh. Compact tunable bandpass filter with wide tuning range of centre frequency and bandwidth using coupled lines and short-ended stubs. IET Microw. Antenna Propag.Vol.10 (2016) No.8, p. 863-870.

[3]. Y.H. Cho, G.M. Rebeiz. Tunable 4-pole noncontiguous $0.7-2.1 \mathrm{GHz}$ bandpass filters based on dual zero-value couplings. IEEE Trans. Microw. Theory Tech. Vol.63(2015) No.5, p. 1579-1586.

[4]. I.Wolff. Microstrip bandpass filter using degenerate modes of a microstrip ring resonator. Electron. Lett.Vol.8(1972)No.12, p.302-303.

[5]. J.S. Hong, M. J. Lancaster. Bandpass characteristics of new dualmode microstrip square loop resonators. Electron. Lett.Vol.31(1995)No.11, p.891-892.

[6]. M. Matsuo, H. Yabuki, M. Makinoto. Dual-mode stepped impedance ring resonator for bandpass filter applications. IEEE Trans. Microw. Theory Tech.Vol.49(2001)No.7, p.1235-1240.

[7]. J.S. Hong, S. Li. Theory and experiment of dual-mode microstrip triangular patch resonators and filters. IEEE Trans. Microw. Theory Tech.Vol.( 2004)No.4,p.1237-1243.

[8]. J.S. Hong, H. Shaman, Y. H. Chun. Dual-mode microstrip openloop resonators and filters. IEEE Trans. Microw. Theory Tech.Vol.55( 2007)No.8, p.1764-1770.

[9]. J.S. Hong, M. J. Lancaster. Couplings of microstrip square openloop resonators for cross-coupled planar microwave filters. IEEE Trans. Microw. Theory Tech.Vol.44(1996)No.12, p.2099-2109.

[10]. Y.H. Chun, J.S. Hong. Electronically Reconfigurable Dual-Mode Microstrip Open-Loop Resonator Filter. IEEE Microwave and Wireless Components Letters. Vol.18 (2008) No.7, p.449-451. 
[11]. X. Wang, Y. Cho, S. Yun. A tunable combline bandpass filter loaded with series resonator. IEEE Trans. Microw. Theory Tech.Vol.60( 2012)No.6, p.1569-1576.

[12]. S. Amari, U. Rosenberg. Adanced coupling matrix synthesis techniques for microwave filters. IEEE Trans. Microw. Theory \& Tech.Vol.51( 2003)No.1, p.1-10. 